\title{
BJO
}

\section{Depression and the vision-related quality of life in patients with retinitis pigmentosa}

B-J Hahm, Y-W Shin, E-J Shim, et al.

Br J Ophthalmol 2008 92: 650-654 originally published online March 20, 2008 doi: 10.1136/bjo.2007.127092

Updated information and services can be found at:

http://bjo.bmj.com/content/92/5/650.full.html

Topic collections Articles on similar topics can be found in the following collections

Eye (globe) (453 articles)

Retina (1005 articles)

Notes

To order reprints of this article go to:

http://bjo.bmj.com/cgi/reprintform

To subscribe to British Journal of Ophthalmology go to:

http://bjo.bmj.com/subscriptions 


\title{
Depression and the vision-related quality of life in patients with retinitis pigmentosa
}

\author{
B-J Hahm, ${ }^{1,2}$ Y-W Shin, ${ }^{1}$ E-J Shim, ${ }^{2}$ H J Jeon, ${ }^{1}$ J-M Seo, ${ }^{3}$ H Chung, ${ }^{3}$ H G Yu ${ }^{3,4}$
}

${ }^{1}$ Department of

Neuropsychiatry, Seoul National

University Hospital, Seoul,

Korea; ${ }^{2}$ Institute of Behavioral

Medicine, Medical Research

Center, Seoul National

University, Seoul, Korea;

${ }^{3}$ Department of Ophthalmology,

Seoul National University,

College of Medicine, Seoul,

Korea; ${ }^{4}$ Sensory Organ Institute,

Medical Research Center, Seoul

National University, Seoul, Korea

Correspondence to:

Professor H G Yu, Department of

Ophthalmology, Seoul National

University College of Medicine,

28 Yongon-dong, Chongno-gu,

Seoul, Korea 110-744;

hgonyu@snu.ac.kr

Accepted 6 February 2008

Published Online First

20 March 2008

\section{ABSTRACT}

Aims: To assess the relationship between depression and the vision-related quality of life in patients with retinitis pigmentosa (RP).

Methods: The study included 144 patients diagnosed as having RP. The mean age of the patients was 38.5 (SD 13.3) years, and $42 \%$ of the subjects were women. They answered the National Eye Institute Visual Function Questionnaire (NEI-VFO) to assess the vision-related quality of life and the Beck Depression Inventory (BDI) to assess depressive symptoms. Patients were classified into groups with and without depression according to the BDI score. The NEI-VFO composite and subscale scores were compared between groups. The correlations between the BDI and the NEI-VFO, weighted visual acuity (WVA) and functional vision score (FVS) were investigated.

Results: The depressed group had significantly less subjective visual function compared with the nondepressed group. A negative correlation was observed between the BDI and the NEI-VFO scores, while no correlation was found between the BDI score and WVA or FVS.

Conclusion: The RP patients with depression had poorer vision-related functions compared with those patients without depression, which cannot be explained by the visual acuity. Interventions to diagnose and treat depression are necessary to enhance the overall quality of life in RP patients.

Of all types of sensory loss, loss of vision is the most severe social and psychological disability, ${ }^{1}$ and retinitis pigmentosa (RP) is a leading cause of inherited blindness among individuals younger than 60 years. ${ }^{23}$ Although the clinical symptoms of RP are highly variable, most patients lose their vision by age 40 , with severely constricted visual fields due to the loss of rod and cone photoreceptor cells. $^{4}$

Visual impairment substantially impairs daily functioning and well-being. ${ }^{5-7}$ Studies report that old people with visual impairment have a higher prevalence of depression compared with people with good vision. ${ }^{6-8} \mathrm{~A}$ 4-year follow-up study of the newly blind reported that the blind patients showed only a minor improvement in the acquisition of compensatory skills, and a significant portion of the patients suffered from depression and overall poor health, with consequent functional impairment. ${ }^{9}$

$\mathrm{RP}$ is emotionally devastating to the patients due to their anticipated vision loss, and the subsequent experience of gradual vision loss causes severe distress. These factors could make RP patients more vulnerable to depression, but few studies have addressed the issues of depression and the vision-related quality of life in this population. Therefore, we assessed the depression status of RP patients in Korea and examined how depressive symptoms affect their subjective visual function and vision-related quality of life.

\section{METHODS}

\section{Subjects}

The patients were recruited through advertisements from the outpatient clinic at Seoul National University Hospital (SNUH) and the Korean Retinitis Pigmentosa Society, a non-profit support network for Korean RP patients. All patients volunteered to participate in a study associated with the Korean Genome Project after being diagnosed by ophthalmological specialists in retinal disease at SNUH. The patients underwent a careful fundus examination and standard electroretinography (ERG) to confirm the diagnosis. Patients with other ocular diseases that could affect visual function were excluded. Initially, 152 patients volunteered for the study, but eight patients were excluded after the ophthalmological examination. Five of them were diagnosed as Stargardt disease, and three patients as choroideraemia. Consequently, 144 patients with RP participated in the study.

\section{Test of visual function}

Best-corrected visual acuity (VA) was measured with Snellen's acuity charts and transformed to the logarithm of the minimum angle of resolution (logMAR). The visual acuity was measured in a standard manner. All the patients were evaluated in the same room in which the degree of illumination was sufficiently bright for measuring visual acuity and not changed. For VA less than counting fingers (CF) at $0.5 \mathrm{~m}$, the following arbitrary $\log M A R$ values were used: CF in front of the eye, $\log M A R$ 2.2; hand movements (HM), logMAR 2.3; light perception (LP), logMAR 2.5; and no LP, logMAR 3. The weighted logMAR visual acuity (WVA) was calculated and used in the analysis. ${ }^{10}$ The monocular and binocular kinetic visual fields were measured with Goldmann perimetry using III/4/e isopter at standard background luminance, and the Binocular Visual Field Score (BVFS) was measured. Since the Functional Vision Score (FVS), which combines the visual acuities and visual fields into one measure, is a better measure of functional vision in daily activity than either VA or VF alone, FVS was also calculated and used in the analysis. ${ }^{11}$ 


\section{Self-reported visual functioning questionnaire}

The National Eye Institute Visual Functioning Questionnaire (NEI-VFQ) was used to evaluate the patients' subjective visual function. ${ }^{12}$ The NEI-VFO-25 gives an overall score, as well as 12 subscale scores: general health, general vision, near vision, distance vision, driving, peripheral vision, colour vision, ocular pain, vision-associated role limitations, dependency, social functioning and mental health. The questionnaire focuses on vision difficulties in everyday life, as well as vision-relevant psychosocial domains, such as mental health, social function and role difficulty. The original questionnaire was translated into Korean separately by two independent clinical experts, a psychiatrist and a psychologist, each with a good command of English. The two versions of the initial translation were checked and combined, and then back translated by a bilingual clinical expert. A four-member translation committee comprising healthcare professionals reviewed and agreed on a version of the final Korean NEI-VFO that best reflected the original, conceptually and linguistically. The patients filled up the package including the questionnaire for demographical information, Beck Depression Inventory (BDI), and NEI-VFQ and their responses were checked at the scheduled visit by a clinician (JMS) and if there was any missing, the patients were asked to fill up the missing. When completion was impossible due to low vision, an interviewer administered it. The questionnaire comprises 5-point scale ratings that were transformed to a 0 100 scale. The overall composite NEI-VFO scores of 11 of the 12 subscales were calculated, excluding the general health subscale. $^{13}$

\section{Beck Depression Inventory}

Depression in the patients with RP was assessed using the BDI, a well standardised 21-item measure of the cognitive, affective and somatic symptoms of depression. The BDI was developed to assess the type and severity of depression based on clinical symptoms, ${ }^{14}$ and is one of the most widely used self-report instruments for measuring the severity of depressive symptoms. The Korean version of the BDI has been standardised formally and a score of 16 was suggested as the optimal cut-off score for the diagnosis of major depression in Korea. ${ }^{15}$ Therefore, in this study, a BDI score $\geqslant 16$ was considered indicative of depression.

\section{Statistical analysis}

The study participants were classified into two groups, a depressed group and a non-depressed group, according to the BDI total score (cut-off point: BDI 16). The demographic profile for age, sex, living arrangements, education level, marital status, socio-economic status ${ }^{16}$ and visual function as measured by the WVA, BVFS, FVS and NEI-VFO composite scores were compared between the patients with and without depression, using the $t$ test and chi-square test for continuous and categorical variables, respectively.

The average NEI-VFO subscale scores between depressed and non-depressed groups were analysed using the test, and the Bonferroni correction was adopted for multiple comparisons. Since the number of current drivers was small (four depressed vs. 17 non-depressed), the driving subscale was excluded from the analysis. Since the violation of the homogeneity of variance between the depressed and non-depressed group can lead to biased results in the analysis with unequal sample size, the Levene's test was performed for equality of variances.
Correlations between the BDI score and NEI-VFQ average score, as well as between the BDI score and the WVA and FVS scores, were examined using Pearson's correlation coefficient. Finally, multiple linear regression analyses were performed to determine predictive factors and their contribution to the NEI$\mathrm{VFO}$ composite score. Covariates included factors that are known to be associated with self-reported visual function: age, sex, socio-economic status, marital status and education level. A $\mathrm{p}$ value $<0.05$ was considered significant in all analyses. Data were analysed with SPSS 12.0 software (SPSS, Chicago).

\section{RESULTS}

The participants were generally young to middle-aged (mean age 38.5 (SD 13.3) years) with an average duration of illness of 18.7 (12.9) years (table 1). Thirty-seven patients (25.7\%) had a BDI score $\geqslant 16$, indicative of clinical depression in the Korean population.

No significant differences were observed between the high and low BDI groups with respect to socio-demographic and clinical variables, including age, sex, education level, living arrangement, socio-economic status, onset age and duration of illness. Although not significant, a trend toward a higher unmarried status was observed in the high BDI group compared with the low BDI group $\left(\chi^{2}=3.26, p=0.071\right)$.

The objective visual function as measured using the WVA, BVFS and FVS showed no significant differences between the high- and low-BDI groups. By contrast, the subjective vision function and related quality of life as measured by the NEI-VFO composite scores differed significantly between the groups. The high-BDI group scored over 15 points lower than the low-BDI group (41.2 (22.6) vs. 56.7 (19.5), $\mathrm{p}<0.001)$. The differences remained significant, even after the vision-specific mental health subscale was excluded from the NEI-VFQ composite score (44.4 (23.4) vs. 58.3 (19.5), p<0.001).

The group with low-BDI scores had higher scores for all of the NEI-VFO subscales, except general and peripheral vision (table 2). All the comparison between depressed and nondepressed group passed the Levene test for equality of variances, except for colour vision $(F=4.6, p=0.03)$. As shown in fig 1 , five subscales remained significant after the Bonferroni correction for multiple comparisons: general health, ocular pain, vision-specific social functioning, vision-specific mental health and vision-specific dependency. The depressed patients experienced the greatest difficulties in the following order: general health, vision-specific mental health and vision-specific role difficulties, followed by general vision activities, distance activities, peripheral vision, vision-specific dependency, near activities, ocular pain and colour vision (fig 1).

Linear regression analysis showed that the BDI and WVA scores were significant predictors of the NEI-VFQ composite score (table 3). The BDI score explained approximately $10.2 \%$ of the variability in the NEI-VFQ composite scores, while the WVA score explained $33.6 \%$ of the variability. The NEI-VFO composite score (vision-specific mental health subscale excluded) was inversely correlated with both the WVA and BDI scores $(r=-0.69,-0.30, p<0.0005)$ (fig 2A, B). By contrast, the BDI was not correlated with any objective measure of visual function, including WVA $(r=-0.01$, $p=0.97)$ or FVS $(r=0.12, p=0.18)$ (fig $2 C, D)$. Other clinical parameters such as age $(r=0.14, p=0.09)$, onset age $(r=0.03$, $p=0.71)$, duration of illness $(r=0.08, p=0.36)$, socio-economic status $(r=0.12, p=0.15)$ or education level $(r=-0.11$, $\mathrm{p}=0.19$ ) showed no correlations with the BDI. 
Table 1 Characteristics of all patients and a comparison of the characteristics of depressed and non-depressed patients with retinitis pigmentosa

\begin{tabular}{|c|c|c|c|}
\hline Characteristics & Total $(n=144)$ & $\mathrm{BDI}<16(\mathrm{n}=107)$ & $B D I \geqslant 16(n=37)$ \\
\hline Age, mean years (SD) & $38.5(13.3)$ & $37.8(13.0)$ & 40.7 (13.9) \\
\hline \multicolumn{4}{|l|}{ Sex, n (\%) } \\
\hline Male & $83(57.6)$ & $64(59.8)$ & $19(51.4)$ \\
\hline \multicolumn{4}{|l|}{ Living arrangements, $\mathrm{n}(\%)$} \\
\hline Living alone & $12(8.3)$ & $9(8.4)$ & $3(8.1)$ \\
\hline Living with someone else & $126(91.7)$ & $92(91.6)$ & $34(91.9)$ \\
\hline Unmarried or divorced & $75(52.1)$ & $51(47.7)$ & $24(64.9)$ \\
\hline Education, mean years (SD) & $12.8(3.8)$ & $13.0(3.7)$ & $12.4(4.2)$ \\
\hline Socio-economic status, mean (SD) & $2.9(1.1)$ & $2.8(1.1)$ & $3.1(1.2)$ \\
\hline Onset age (years), mean (SD) & $19.3(14.0)$ & $20.0(13.6)$ & $17.3(15.0)$ \\
\hline Duration of illness, mean (SD & $18.7(12.9)$ & $17.6(12.1)$ & $22.3(14.7)$ \\
\hline NEI-VFO-25, mean (SD) & $52.7(21.4)$ & $56.7(19.5)$ & $41.2(22.6)^{*}$ \\
\hline NEI-VFO-25, mean (SD) (mental subscale not included) & $51.9(20.9)$ & $58.3(19.5)$ & $44.4(23.4)^{*}$ \\
\hline
\end{tabular}

${ }^{*} \mathrm{p}<0.05$.

BDI, Beck Depression Inventory; NEI-VFQ, National Eye Institute Visual Function Questionnaire.

\section{DISCUSSION}

We examined depression and its impact on RP patients' perception of their vision function and related quality of life. The results show that depression is common in RP patients and that the subjective visual function and vision-related quality of life are affected by the severity of depression, as well as objective visual disturbance. The prevalence of depression in the study was $25.7 \%$, much higher than the $10 \%$ prevalence in the general population ${ }^{6}$ but similar to the rates found in studies of other chronic disease such as diabetes, ${ }^{17}$ postmyocardial infarction ${ }^{18}$ and cancer. ${ }^{19}$

We found that the RP patients with depression had significantly lower self-reported vision function compared with those without depression. In addition, the depression in RP patients had a strong negative correlation with the self-reported

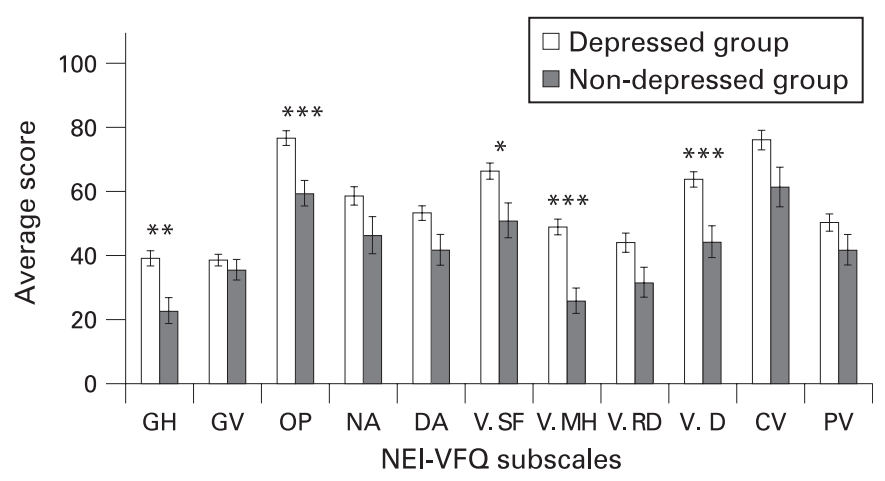

Figure 1 National Eye Institute Visual Function Questionnaire (NEIVFO) subscale scores for the depressed and non-depressed patients with retinitis pigmentosa. $\mathrm{BDI}$, Beck Depression Index; CV, colour vision; DA, distance activities; GH, general health; GV, general vision; NA, near activities; OP, ocular pain; PV, peripheral vision; V.D, vision-specific dependency; V.MH, vision-specific mental health; V.RD, vision-specific role difficulties; V.SF, vision-specific social functioning. A higher score represents a better function in the corresponding subscale. * Significance at the Bonferroni-adjusted (0.05/11) 0.0045 level (two-tailed).

${ }^{* *} p<0.0009(0.01 / 11)$. ${ }^{* *} p<0.0004(0.005 / 11)$. vision function, while no such associations were found with objective visual function measures such as the WVA or FVS and the clinical parameters like age, age at onset or duration of illness. Therefore, the results indicate that the severity of depression is not influenced by the progression of RP, and depression affects self-reported vision function, independent of the objective disease status of RP. This is consistent with previous findings in a community population that depression increases the likelihood of vision-related functional impairment independent of vision impairment. ${ }^{8}$

The impact of depression on the patient-reported vision function was substantial. The average NEI-VFO composite score in the patients without depression was higher than in the patients with depression, with a difference of about 15 points. This difference in the NEI-VFO composite score was larger than the difference between the patients with unilateral and bilateral visual impairment. ${ }^{20}$ Miskala et al reported that 3.6- to 16.2point decreases in the composite NEI-VFO score corresponded to a three-line decrease in the visual acuity of the better-seeing eye. ${ }^{21}$ The depressed group had a lower NEI-VFO average score for all subscales except general and peripheral vision. This suggests that the effect of depression on the vision-related quality of life is pervasive in patients with RP.

Five subscales showed sufficiently large differences between the depressed and non-depressed groups to survive the multiple comparisons: vision-specific mental health, vision-specific social function, vision-specific dependency, general health and ocular pain. The five subscales share a common feature in that they measure relatively subjective aspects of vision-related functions compared with the other subscales. For example, a large difference was found in the general health subscale, while no difference was observed in the general vision subscale. The general health and general vision subscales each comprise one question to evaluate the subject's "overall health" and "eyesight," respectively. The "overall health" is more subjective and is a broader term than "eyesight," which has a narrower connotation. Similarly, the vision-specific social function reflects subjective judgement of their visual impairment, unlike the subscales that evaluate specific symptomatology as in the 
Table 2 National Eye Institute Visual Function Questionnaire subscale scores for the depressed and non-depressed patients with retinitis pigmentosa

\begin{tabular}{llrl}
\hline $\begin{array}{l}\text { National Eye Institute Visual Function } \\
\text { Questionnaire subscores }\end{array}$ & $\mathbf{B D I}<\mathbf{1 6}$ (n= 107) & $\mathbf{B D I} \geqslant \mathbf{1 6}$ (n= 37) \\
\hline General health** & $39.3(24.3)$ & $23.0(24.6)$ & $<0.001$ \\
General vision & $38.7(18.9)$ & $35.7(19.5)$ & 0.408 \\
Ocular pain*** & $76.8(23.4)$ & $59.5(24.4)$ & $<0.001$ \\
Near activities & $58.7(29.6)$ & $46.4(35.2)$ & 0.039 \\
Distance activities & $53.4(23.4)$ & $41.9(29.2)$ & 0.017 \\
Vision-specific social functioning* & $66.5(25.7)$ & $51.0(32.9)$ & 0.004 \\
Vision-specific mental health** & $49.1(25.0)$ & $26.0(24.0)$ & $<0.001$ \\
Vision-specific role difficulties & $44.2(30.9)$ & $31.8(28.2)$ & 0.033 \\
Vision-specific dependency*** & $64.0(24.5)$ & $44.4(30.0)$ & $<0.001$ \\
Colour vision & $76.2(31.2)$ & $61.5(37.5)$ & 0.037 \\
Peripheral vision & $50.5(27.9)$ & $41.9(28.9)$ & 0.112
\end{tabular}

\footnotetext{
${ }^{*}$ Significance at the Bonferroni-adjusted (0.05/11) 0.0045 level (two-tailed).

${ }^{* *} p<0.0009(0.01 / 11)$.

${ }^{* * *} p<0.0004(0.005 / 11)$.

BDI, Beck Depression Inventory.
}

subscales of near-vision or peripheral-vision subscales. The depressed patients scored an average of 60 points in the ocular pain subscale, corresponding to mild to moderate levels of pain or discomfort in and around the eyes, while the patients without depression had no or mild levels of discomfort. This finding is comparable with many previous reports that depression reduces the pain perception threshold ${ }^{22}$ and implies that depression might aggravate or induce ocular pain in patients with RP.

Indirect evidence exists that depression in patients with RP can be treated successfully, and the vision-related function can be improved. Brody et al found that self-management education programme caused $50 \%$ reduction in incidence of depression at 6-month follow-up in the patients with advanced macular
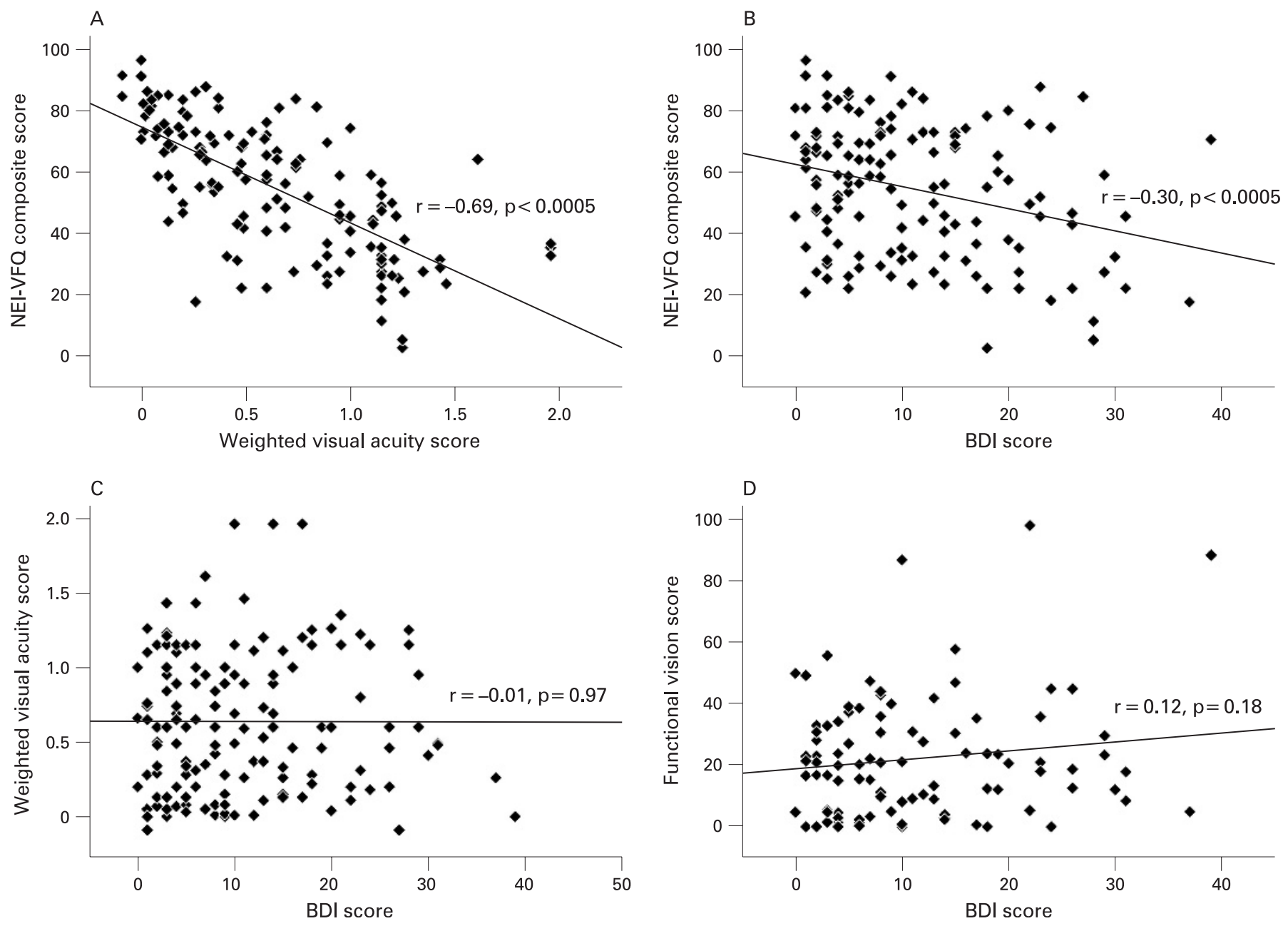

Figure 2 Four correlations: between the National Eye Institute Visual Function Questionnaire (NEI-VF0) composite score and the Beck Depression Inventory (BDI) and weighted visual acuity (WVA) scores, and between the BDI score and the WVA and functional vis scores. 
Table 3 Summary of the linear regression model between the National Eye Institute Visual Function Questionnaire composite score and the scores for the Beck Depression Inventory, weighted visual acuity and other clinical parameters

\begin{tabular}{lrrr}
\hline Variables & \multicolumn{1}{l}{$\boldsymbol{\beta}$} & \multicolumn{1}{l}{$\mathbf{p}$} \\
\hline Sex & 0.006 & 0.090 & 0.928 \\
Age & -0.021 & -0.245 & 0.807 \\
Duration of illness & -0.085 & -1.158 & 0.249 \\
Beck Depression & -0.329 & -5.169 & $<0.001$ \\
Inventory & & & \\
Weighted visual acuity & -0.644 & -9.475 & $<0.001$ \\
Marital status & -0.103 & -1.508 & 0.134 \\
Education & -0.030 & -0.415 & 0.679 \\
Socio-economic status & -0.009 & -0.128 & 0.898 \\
\hline
\end{tabular}

degeneration. ${ }^{23}$ Recently, Rovner et al reported that short-term psychological treatment aimed at increasing problem-solving skills lowered the incidence of depression and increased visionrelated function as measured by the NEI-VFO-17. ${ }^{24}$

Our study has several important limitations. We did not diagnose the depression using conventional diagnostic criteria, but instead measured the symptoms of depression in RP patients using a self-reported questionnaire, the BDI. Note that although the BDI is not a diagnostic tool for depression, the BDI has been confirmed to be a good screening method for depression. ${ }^{25}$ Because we included only volunteers, we cannot exclude the possibility that the patients with more severe depression did not volunteer as often as the patients with less severe depression, which may underestimate the prevalence of depression in RP patients. Moreover, all of the participants were Korean RP patients, and comparable studies in other countries would be useful to test the generalisability of our results.

In conclusion, this study showed that RP patients are at increased risk of depression, which reduces the patients' visionrelated quality of life. Depression was found to be a unique contributing factor influencing the vision-related quality of life, independent of demographic status, objective vision function and disease severity of RP. As RP is a lifelong disease without treatment options, the supportive management of vision-related symptoms is critical for RP patients. Our findings suggest that depression should be assessed in RP patients and that the patients may benefit from interventions targeting depression, which negatively affects their vision-related quality of life.

Acknowledgements: Drs Shin and Hahm contributed equally to this article. We thank M-S Kim, who provided great help in recruiting the patients and gathering data for the study.

Competing interests: None.
Patient consent: All subjects signed an informed consent form approved by the institutional review board.

\section{REFERENCES}

1. Greenberg G. Beyond the blindfold: my life with retinitis pigmentosa. Clin Dermatol 2005:23:640-2; discussion 642-3.

2. Al-Merjan JI, Pandova MG, Al-Ghanim M, et al. Registered blindness and low vision in Kuwait. Ophthalmic Epidemiol 2005;12:251-7.

3. Buch H, Vinding T, La Cour M, et al. Prevalence and causes of visual impairment and blindness among 9980 Scandinavian adults: the Copenhagen City Eye Study. Ophthalmology 2004;111:53-61.

4. Hartong DT, Berson EL, Dryja TP. Retinitis pigmentosa. Lancet 2006;368:1795-809.

5. Salive ME, Guralnik J, Glynn RJ, et al. Association of visual impairment with mobility and physical function. J Am Geriatr Soc 1994;42:287-92.

6. Evans JR, Fletcher AE, Wormald RP. Depression and anxiety in visually impaired older people. Ophthalmology 2007;114:283-8.

7. Carabellese C, Appollonio I, Rozzini R, et al. Sensory impairment and quality of life in a community elderly population. J Am Geriatr Soc 1993;41:401-7.

8. Rovner BW, Ganguli M. Depression and disability associated with impaired vision: the MoVies Project. J Am Geriatr Soc 1998;46:617-9.

9. Fitzgerald RG, Ebert JN, Chambers M. Reactions to blindness: a four-year follow-up study. Percept Mot Skills 1987;64:363-78.

10. Holladay JT. Proper method for calculating average visual acuity. J Refract Surg 1997;13:388-91.

11. Fuhr PSW, Holmes LSD, Flecher DC, et al. The AMA Guides functional vision score is a better predictor of vision-targeted quality of life than traditional measures of visual acuity or visual field extent. Vis Impair Res 2003;5:137-46.

12. Mangione CM, Lee PP, Pitts J, et al. Psychometric properties of the National Eye Institute Visual Function Questionnaire (NEI-VFO). NEI-VFO Field Test Investigators. Arch Ophthalmol 1998;116:1496-504.

13. Mangione CM, Lee PP, Gutierrez PR, et al. Development of the 25-item National Eye Institute Visual Function Questionnaire. Arch Ophthalmol 2001;119:1050-8.

14. Beck AT, Ward $\mathrm{CH}$, Mendelson $\mathrm{M}$, et al. An inventory for measuring depression. Arch Gen Psychiatry 1961;4:561-71.

15. Shin M, Kim Z, Park K. The cut-off score for the Korean version of Beck Depression Inventory. Korean J Clin Psychol 1993;12:71-81.

16. Hollingshead AB, Redlich FL. Social class and mental illness. New York: Wiley, 1958.

17. Anderson RJ, Freedland KE, Clouse RE, et al. The prevalence of comorbid depression in adults with diabetes: a meta-analysis. Diabetes Care 2001;24:106978.

18. Kaptein KI, de Jonge $\mathrm{P}$, van den Brink RH, et al. Course of depressive symptoms after myocardial infarction and cardiac prognosis: a latent class analysis. Psychosom Med 2006;68:662-8.

19. Massie MJ. Prevalence of depression in patients with cancer. J Nat/ Cancer Inst Monogr 2004;32:57-71.

20. Varma R, Wu J, Chong K, et al. Impact of severity and bilaterality of visual impairment on health-related quality of life. Ophthalmology 2006;113:1846-53.

21. Miskala PH, Hawkins BS, Mangione CM, et al. Responsiveness of the National Eye Institute Visual Function Questionnaire to changes in visual acuity: findings in patients with subfoveal choroidal neovascularization-SST Report No. 1. Arch Ophthalmol 2003;121:531-9.

22. Chiu YH, Silman AJ, Macfarlane GJ, et al. Poor sleep and depression are independently associated with a reduced pain threshold. Results of a population based study. Pain 2005;115:316-21.

23. Brody BL, Roch-Levecq AC, Thomas RG, et al. Self-management of age-related macular degeneration at the 6-month follow-up: a randomized controlled trial. Arch Ophthalmol 2005;123:46-53.

24. Rovner BW, Casten RJ, Hegel MT, et al. Preventing depression in age-related macular degeneration. Arch Gen Psychiatry 2007;64:886-92.

25. Bennett DS, Ambrosini PJ, Bianchi M, et al. Relationship of Beck Depression Inventory factors to depression among adolescents. J Affect Disord 1997;45:127-34. 